\title{
Iron and Atherosclerosis: Nailing Down a Novel Target with Magnetic Resonance
}

\author{
Travis P. Sharkey-Toppen, MS, Arun K. Tewari, PhD, and Subha V. Raman, MD, MSEE \\ The Ohio State University, Columbus, Ohio
}

\begin{abstract}
Iron is an essential mineral in many proteins and enzymes in human physiology, with limited means of iron elimination to maintain iron balance. Iron accrual incurs various pathological mechanisms linked to cardiovascular disease. In atherosclerosis, iron catalyzes the creation of reactive oxygen free radicals that contribute to lipid modification, which is essential to atheroma formation. Inflammation further fuels iron-related pathologic processes associated with plaque progression. Given iron's role in atherosclerosis development, in vivo detection techniques sensitive iron are needed for translational studies targeting iron for earlier diagnosis and treatment. Magnetic resonance imaging (MRI) is uniquely able to quantify iron in human tissues noninvasively and without ionizing radiation, offering appealing for longitudinal and interventional studies. Particularly intriguing is iron's complementary biology vs. calcium, which is readily detectable by computed tomography (CT). This review summarizes the role of iron in atherosclerosis with considerable implications for novel diagnostic and therapeutic approaches.
\end{abstract}

\begin{abstract}
Atherosclerosis is a proliferative, inflammatory disease affecting the arteries. Plaque disruption and subsequent thrombosis from the exposure of plaque contents to circulating blood is the underlying cause of acute coronary syndromes and ischemic strokes. Current preventive strategies focus on management of traditional risk factors such as dyslipidemia, diabetes, hypertension and smoking, and current treatment strategies of more advanced disease employ mechanical relief of stenosis in conjunction with lipid-lowering, anti-platelet and anti-ischemic medications. Yet, despite significant reduction of morbidity and mortality afforded by these strategies, atherosclerosis persists as a leading cause of death worldwide [1].

Atherosclerosis may develop insidiously prior to causing clinically-apparent events like heart attack and stroke; reducing such events requires better mechanistic understanding of disease onset and progression, and improved identification and treatment of subclinical disease. The ability to detect subclinical arterial wall disease holds promise for more timely diagnosis, more effective therapies and better outcomes, particularly if based on a proven contributor to atherosclerosis that also can be measured in vivo. This review focuses on iron as both a biomarker and biomechanistic contributor to the pathogenesis of atherosclerosis. There is now considerable evidence supporting iron's role in both atherosclerosis initiation
\end{abstract}

Correspondence: Subha V. Raman, MD, MSEE The Ohio State University Davis Heart and Lung Research Institute 473 W. $12^{\text {th }}$ Ave, Suite 200 Columbus, OH 43210 phone 614 293-8963 fax 614 293-5614 raman.1@ osu.edu. 
and progression (Figure 1), plus recent clinical trial evidence that targeting iron excess in atherosclerosis may be beneficial. This review summarizes i) evidence supporting iron's role in atherosclerosis from in vitro, animal model and clinical studies (Tables 1 and 2), ii) implications for earlier and more predictive diagnosis and iii) opportunities for future translational research to improve atherosclerosis death and disability by targeting this relatively underappreciated contributor to the disease.

\section{Iron Homeostasis and Its Regulation}

\section{Iron Balance}

Gut absorption is the only source of input to iron balance. Both men and women shed 14 $\mu \mathrm{g} / \mathrm{kg} / \mathrm{d}$ of iron through epithelial sloughing [2]. In addition, premenopausal women lose the daily equivalent of $0.9 \mathrm{mg}$ of iron through menses [3]. Women's levels of ferritin rise dramatically in the fifth and sixth decades of life, typically when menopause occurs, whereas men experience their greatest increase in ferritin levels during the late teenage years [4]. Iron excess may occur via augmented dietary or supplemental intake [5], genetic disorders such as certain forms of hemochromatosis that lead to excess intestinal iron absorption [6] or transfusion-induced iron overload [7]. Iron deficiency most commonly occurs through blood loss but may also ensue from inadequate dietary intake, dietary inhibitors [8], states of increased dietary iron requirement such as pregnancy [9] or conditions such as celiac disease with abnormal gastrointestinal tract mucosa leading to iron malabsorption [10].

\section{Transport, Storage and Regulation}

Free iron ions are toxic due to their redox activity. Therefore, the vast majority of iron in the body exists in bound form. Approximately $2500 \mathrm{mg}$ of iron is in erythrocyte hemoglobin, $1000 \mathrm{mg}$ is bound within ferritin in specific storage tissues (e.g., liver, spleen), $170 \mathrm{mg}$ is in skeletal muscle myoglobin and $3 \mathrm{mg}$ circulates in the transport protein transferrin [11].

Hepcidin is primarily produced by the liver and is the key iron-regulating hormone [12-16]. Hepcidin acts by binding and downregulating ferroportin [17], the essential transmembrane protein for cellular iron export. On the surface of macrophages, ferroportin allows egress of iron. On the basolateral membrane of absorptive intestinal enterocytes, ferroportin facilitates absorption of dietary iron [18-20]. Thus, hepcidin promotes i) increased iron sequestration in macrophages and hepatocytes and ii) decreased intestinal iron absorption by enterocytes. Patient with hemochromatosis, notably due to mutations in TFR2, have extremely low hepcidin levels causing little to no iron availability in macrophages [21]. Lack of macrophage iron to promote atheroma formation could then explain their relative protection from atherosclerosis despite their iron overload status.

Hepcidin is inhibited by anemia and hypoxia, freeing iron for erythropoiesis [22]. During iron loading and inflammation, hepcidin is stimulated by an iron feedback mechanism [22-24]. A similar state is seen in anemia of chronic disease, where patients have a relative deficiency of iron because it is trapped in macrophages. Levels of circulating cytokines including interleukin-6 (IL-6), which stimulates hepcidin expression, are markedly elevated in this chronic inflammatory state [25-27]. Elevated hepcidin has also been associated with 
presence and number of metabolic syndrome features [28] and arterial stiffness by anklebrachial pulse wave velocity [29]. In chronic hemodialysis patients, associations were found between hepcidin-25 levels and increased high sensitivity C-reactive protein, decreased glomerular filtration rate and presence of diabetes mellitus [30]. Galesloot et al. demonstrated after multivariable regression an association between increased quartiles of hepcidin and abnormal carotid intima media thickness (CIMT) or ankle-brachial index (ABI) in post-menopausal females; the association with $\mathrm{ABI}$ was also significant in men [31].

\section{Inflammation and Iron}

\section{Macrophages}

Immunologically-active macrophages are central to the understanding, diagnosis, and treatment of atherosclerosis [32]. Lipid-overloaded macrophages known as foam cells are key constituents of the developing atheroma [32]. Under physiological conditions, ATP binding cassette transporter subfamily 1 (ABCA1), also known as the cholesterol efflux regulatory protein, mediates the efficient efflux of scavenged cholesterol from macrophages to lipid-poor apolipoprotein A-I (apoA-I). Further efflux of cholesterol and lipidation of nascent apoA-I is mediated by ABCG1 and ABCG4 [33]. The resulting high density lipoprotein (HDL) is transported back to the liver via plasma, completing the multi-step process of reverse cholesterol transport (RCT) [34]. The upregulation of ABCA1 and ABCG1 by the administration of a bone morphogenetic protein inhibitor has been shown to decrease the formation of foam cells in atherosclerotic plaque in mice, while concurrent administration of exogenous hepcidin reverses this effect [35]. A particularly intriguing study by Koeth et al. showed that increased red meat intake raised plasma L-carnitine levels that, in turn, correlated with increased atherosclerosis events beyond traditional risk predictors[36].These data support the role of impaired RCT and iron homeostasis in mediating the effects of transporters leading to the abundance of foam cells in atherosclerotic plaque.

\section{Iron-induced oxidative damage}

Electron transfer between iron ions is essential in the transportation, use and storage of iron. Redox-active iron catalyzes the generation of free radicals capable of oxidation of other molecules and proteins such as low-density lipoprotein (LDL)[37]. This iron-catalyzed free radical production is summarized in the Haber-Weiss reaction and Fenton chemistry.

One of the main mechanisms involved in iron-induced oxidative stress is free radical formation. The redox-active ferrous form of iron $\left(\mathrm{Fe}^{2+}\right)$ is primarily responsible for generating highly reactive hydroxyl radicals that, in turn, contribute to the formation of insoluble fibrin clot from modifications of soluble fibrinogen [38]. Scavenger receptors on the surface of macrophages, most notably SR-A and CD36, bind and internalize oxidized LDL (oxLDL). Endocytosed oxLDL upregulates CD36 expression by activation of PPAR $\gamma$ transcription factor, increasing macrophage sensitivity to oxLDL scavenging [39] but also enhancing transcription of ABCA1 for increased cholesterol efflux [40, 41]. However, oxLDL accumulated in this manner tends to become trapped in macrophages forming foam 
cells, consistent with a failure in the efflux mechanism. Iron-induced oxidation of LDL was recently shown to occur in the low $\mathrm{pH}$ environment of intra-macrophage lysosomes [42]. In showing that a-tocopherol enrichment of LDL initially increased the rate of LDL oxidation, this work also offers a mechanistic explanation for the failure of vitamin $\mathrm{E}$ to reduce atherosclerotic events in clinical trials.

Given the heightened atherosclerosis risk born by patients with diabetes mellitus, it is worth noting that i) increased dietary iron intake increases diabetes risk[43] and ii) haptoglobin gene variants that impair iron clearance are associated with greater intraplaque iron in diabetic patients[44]. Thus, consideration of means to reduce atherosclerotic burden by targeting iron may be particularly important for these high-risk patients.

\section{Magnetic Resonance Imaging of Iron and Atherosclerosis}

Detection of subclinical atherosclerosis typically involves imaging, noting that modalities such as stress testing are only abnormal in the face of advanced plaque producing stenosis of the arterial lumen. Catheterization-based imaging techniques like intravascular ultrasound, optical coherence tomography and other invasive approaches can certainly identify and characterize plaque. However, the costs and risks inherent to such tests limit their appeal for asymptomatic individuals seeking identification of subclinical disease to guide treatment strategies. Noninvasive arterial ultrasonography holds some appeal in detecting arterial wall thickening [45] and vasa vasorum proliferation [46]. Limited spatial imaging depth, however, limits the extent to which this modality may characterize atherosclerosis-prone vessel walls beyond the readily-accessible carotid arteries.

Magnetic resonance imaging (MRI) is an established, high-resolution tool for in vivo imaging of the carotid arteries and atherosclerotic plaque using multi-contrast signal based techniques such as T1-weighted (T1w), T2-weighted (T2w), proton density and time-offlight imaging [47-53]. Several studies have demonstrated the ability to differentiate calcification $[47,48,54,55]$, the fibrous cap [49, 50, 54-56] and lipid-rich necrotic core (LRNC) [47, 48, 51, 55-58] on T1-weighted (T1w) images, and even the presence of intraplaque hemorrhage (IPH) [47-49, 52-55, 59-63] by multispectral analysis of T1w and timeof-flight (TOF) images. These methods have also been successfully applied to other major arteries such as the aorta [64] and femoral arteries [65]. Beyond its enhanced ability to characterize soft tissues when compared to other imaging modalities, MRI is uniquely suited to measure iron in tissues [66-68]. The impact of MRI-based tissue iron quantification cannot be overstated: MRI-based measurement of myocardial iron content has led to a $71 \%$ reduction in mortality in patients at risk of cardiac iron overload [69]. The potential for similar impact in patients at risk of atherosclerosis via arterial iron quantification behooves consideration of the paramagnetic properties of iron that make MRI-based iron detection possible.

\section{Paramagnetic Iron}

In a magnetic field, radiofrequency energy applied at the appropriate resonant frequency causes suitable nuclei in tissues to move from their equilibrium positions; subsequent removal of such energy allows the nuclei to return to their original positions at characteristic 
relaxation rates. Magnetic field inhomogeneities affect the observed relaxation rates. Such inhomogeneities are defined as imperfections in the main magnetic field, chemical shifts, gradient encodings, and susceptibility differences between tissues. So the observed transverse relaxation time or $\mathrm{T} 2 *$ ( $\mathrm{T}$ two star) is a combination of the intrinsic relaxation of the given tissue, $\mathrm{T} 2$, and the added dephasing effects of the inhomogeneity or $\mathrm{T} 2^{\prime}$. This relationship is described $1 / \mathrm{T} 2 *=1 / \mathrm{T} 2+1 / \mathrm{T} 2^{\prime}$ or $1 / \mathrm{T} 2 *=1 / \mathrm{T} 2+\gamma \Delta \mathrm{B}_{\text {inhomogeneity }}$, where $\gamma$ is the gyromagnetic ratio and $\Delta \mathrm{B}_{\text {inhomogeneity }}$ is the magnetic field inhomogeneity present in the observed voxel [70].

Of particular interest to the study of tissue iron content using MRI are the inhomogeneities caused by the positive magnetic susceptibility of iron. Dependent on its quantity and configuration, iron is considered paramagnetic, superparamagnetic or even ferromagnetic. At normal body temperatures, ferritin is paramagnetic with a susceptibility of $1.6 \times 10^{-6}$ $\mathrm{m}^{3} / \mathrm{kg}_{\mathrm{Fe}}$ [71]. This means that when magnetized such as during an MRI, iron deposits will form their own local magnetic field, thus contributing to the observed changes in the overall B field, $\Delta \mathrm{B}_{\text {inhomogeneity. }}$ Compare this to most tissues in the body made up mostly of water with a negative susceptibility or even other trace metals such as calcium which are slightly positive and effect a negligible susceptibility difference [72]. The result is that at longer echo times, images generated by a gradient echo MRI technique will have more rapidly decaying signal anywhere iron is clustered. The resulting hypointense areas can be appreciated qualitatively, or T2* at a specific tissue location (e.g. myocardium, liver, arterial wall) may be estimated by fitting the signal decay over multiple echoes at that location [67, 73].

\section{Endogenous Iron Imaging}

$\mathrm{T} 2 *$ quantification captures local magnetic field inhomogeneity that, in biologic tissues, is most significantly affected by iron. This has primarily been used to date for accurate estimate of tissue iron content in the liver and myocardium [66-68] with Kirk et al. showing an interstudy reproducibility (expressed as the coefficient of variation) of 5.8\% and 5.9\%, respectively [74]. Although, our group and others [75] have documented the presence of iron in atherosclerotic plaques (Figure 2). In carotid atherosclerosis disease, our group has refined this technique to show that intraplaque $\mathrm{T} 2 *$ quantification reflects micro-hemorrhage and iron deposition, with more abnormal $\mathrm{T} 2 *$ found in patients with symptom-producing plaques [76] (Figure 3). We are unaware if these techniques have been applied to peripheral artery disease (PAD) or aortic atherosclerotic plaques, but it would be reasonable to expect similar results in relatively superficial vessels with PAD like the superficial femoral artery or in large enough plaques of the aorta.

\section{Exogenous Iron Imaging}

Arterial wall T2* quantification has also been used to quantify macrophage uptake of ultrasmall paramagnetic iron oxide (USPIO) contrast media to measure inflammatory burden in atherosclerotic disease [77] and response to statin therapy [78]. This contrast takes advantage of the iron sequestration of macrophages, which is enhanced during inflammation by the up regulation of divalent metal transporter-1 (DMT-1) expression by interferon- $\gamma[26$, $79,80]$. 


\section{Iron vs. Calcium in Atherosclerosis}

Given that calcification is a prevalent component of atherosclerotic plaque plus the ease and high resolution of computed tomography (CT) for in vivo imaging of arterial calcification, calcium imaging has garnered widespread acceptance as a means for noninvasive atherosclerosis detection [81-83]. CT performs well in quantifying calcium even in the coronary arteries, and the coronary calcium score has consistently performed well in longitudinal studies of cardiovascular event risk [84, 85]. However, individual plaques have been shown to be more likely to cause events if they are noncalcified vs. calcified [86]. Rajendran et al. in a rabbit model of atherosclerosis offer some insight as to why iron vs. calcium may help explain this apparent paradox [87]. With meticulous mapping of plaque sections using various ion beam techniques, they found an inverse relationship in the distributions of iron and calcium. When interpreted in the context of observational studies in patients, these intriguing data suggest that calcium may defend against plaque destabilization by excluding intra-lesion iron that is more likely to contribute to eventproducing plaque disruption.

Further translational studies are needed to prove that iron, not calcium, is responsible for plaque destabilization in humans, and MRI is an appealing technique to generate the necessary evidence. Calcifications within atherosclerotic plaque appear as hypointense areas on T2-weighted imaging. Other MRI methods of differentiation calcium from iron include ultrashort echo time gradient echo imaging [88] and susceptibility weighted imaging that has already been used to differentiate iron and calcium within the brain [89]. The noninvasive nature of MRI, lack of exposure of human subjects to ionizing radiation and the intrinsic signal of iron that allows MRI-based detection without exogenous contrast administration make MRI an appealing translational approach to further elucidate iron's role in human atherogenesis and plaque progression.

\section{Current Guidelines}

The ultimate clinical impact of MRI-based plaque iron quantification requires demonstration of incremental value over current atherosclerosis management. In this section, we focus on the carotid atherosclerosis to illustrate current guidelines for diagnosis and management.

\section{Diagnosis}

Carotid auscultation affords bedside detection of carotid artery atherosclerosis that produces turbulent flow, which may be minimal in the absence of significant stenosis or in the setting of critical stenosis. Ultrasonography (US) has sufficient sensitivity to measure the combined thickness of the intima and media of the arterial wall, and C-IMT has gained some acceptance as a suitable biomarker of subclinical disease in asymptomatic adults at intermediate risk of atherosclerosis [90]. Despite known limitations in measurement accuracy and reliability [91, 92], clinical trials have relied on C-IMT as an outcome measure [93].

There is greater consensus regarding the use of carotid US in patients with known or suspected carotid stenosis [94], which is advocated for asymptomatic patients with 
significant risk factors, symptomatic atherosclerotic disease in other arterial segments or other to evaluate a bruit detected by auscultation. US may indicate significant stenosis by either direct visualization of a narrowed lumen or by demonstration of flow acceleration through a stenotic site [95]. Noninvasive angiographic techniques including magnetic resonance (MRA), computed tomography (CTA) and invasive catheter angiography are available when i) US findings are equivocal or nondiagnostic or ii) intervention for carotid stenosis is being planned.

\section{Treatment}

Just as diagnostic techniques focus on detecting presence and severity of carotid artery stenosis, treatment algorithms focus on percent luminal stenosis in addition to the usual recommendations for risk factor management. Notably, presence or absence of symptoms attributable to internal carotid artery (ICA) disease dictates the stenosis threshold for intervention. Revascularization, which encompasses surgical carotid endarterectomy and carotid stenting, is advised for symptomatic patients with $>70 \%$ stenosis by noninvasive imaging or $>50 \%$ ICA stenosis by invasive angiography, and is deemed 'reasonable' for asymptomatic patients with $>70 \%$ stenosis of the ICA [94]. The benefit of CEA is significant for patients who survive the 30-day perioperative period (underscoring the need to have such procedures done at centers where the perioperative stroke and mortality rate is $<6 \%$ ) and realize a relatively low risk of ipsilateral stroke of 0.5 to $1.1 \%$ per year.

\section{Limitations of Current Approaches}

Current diagnostic modalities such as duplex ultrasonography, CTA, MRA and catheter angiography perform well in identifying stenotic carotid artery disease, and considerable stroke risk reduction is realized for appropriate patients with high-grade luminal stenosis who undergo revascularization. However, these techniques do not address the composition of plaque that has been shown to predict likelihood of subsequent events [96]. Prospective studies of asymptomatic patients with mildly stenotic (50-79\%) carotid plaques showed the presence of intraplaque hemorrhage, thin fibrous cap, large LRNC and maximal wall thickness on MRI were all significantly correlated with subsequent cerebrovascular events [96]. Retrospective studies of ruptured plaques that produced acute coronary events have shown that $78 \%$ of culprit lesions had $<70 \%$ luminal stenosis, falling below current threshholds for revascularization had the plaque been detected prior to the clinical event [97].

\section{Translational Approaches Advancing Iron's Role in Atherosclerosis}

Given considerable mechanistic and observational data supporting iron's role in atherosclerosis, several groups have assessed the effect on iron by existing antiatherosclerotic therapies or novel approaches that target iron.

\section{Statin therapy}

While lipid-lowering therapy is already recommended for all patients with extra cranial carotid atherosclerosis to reduce low-density lipoprotein cholesterol below $100 \mathrm{mg} / \mathrm{dL}$ [94], there is evidence to suggest that the pleiotropic effects of statins impact iron metabolism. In 
particular, statin therapy appears to downregulate hepcidin, leading to decreased degradation of ferroportin and increased efflux of iron from macrophages [98]. Zacharski et al., whose randomized trial of iron reduction via phlebotomy in patients with established peripheral arterial disease showed variable impact on outcomes in all patients[99], recently published secondary outcome results focused on statin therapy [100]. In this analysis, reduction in allcause mortality with statins paralleled lowering of ferritin levels but not improvement in lipid profiles.

\section{Chelation}

The chelating agent deferoxamine (DFO) has been shown to reduce lesion size in atheromaprone rabbits [101] and improve endothelium-dependent vasodilation in patients with coronary artery atherosclerosis [102]. The recently published Trial to Assess Chelation Therapy evaluated in a double-blind, placebo-controlled randomized study design the impact of intravenous disodium EDTA in patients with a history of myocardial infarction[103]. In this population with established coronary atherosclerosis, chelation demonstrated a modest reduction in risk of adverse cardiovascular outcomes.

\section{Other potential therapeutic targets}

Heme-oxygenase-1 (HO-1) has been shown to play an important role in preventing vascular inflammation. In response to pro-atherogenic signals, HO-1 expression is increased leading to release of ferrous iron from heme. Given its known antioxidant, anti-apoptotic, antiproliferative properties, $\mathrm{HO}-1$ is being investigated as a novel therapeutic target in atherosclerosis [104]. Increased levels of hepcidin results in iron sequestration in macrophages, making it readily available for lipid peroxidation. Availability of anti-hepcidin antibodies [105] should facilitate further development of anti-atherogenic approaches targeting hepcidin's interactions with iron and macrophages in the plaque microenvironment.

\section{Future Directions}

Considerable evidence has accrued supporting iron as a mechanistic contributor to this disease, and unique aspects of iron's biology in tissues make it appealing as both a diagnostic and therapeutic target. While older epidemiological studies failed to find an association between conventional blood iron levels and atherosclerotic events[106], contemporary approaches incorporating more refined measures do support a connection between iron homeostasis and atherosclerosis that is consistent with the accruing mechanistic evidence. Efficient pharmacological manipulation of iron homeostasis requires careful consideration of the essential role that iron plays in a considerable number of physiological pathways while recognizing opportunities to influence iron's pathologic role in atherogenic processes. As atherosclerosis remains a leading cause of death and disability, further translational studies evaluating iron and atherosclerosis are warranted.

\section{Acknowledgments}

Grant support: 5R01HL95563 (SVR) 
Relationships with Industry: Siemens (SVR)

\section{Literature Cited}

1. World Health Organization. World Health Statistics. World Health Organization; Geneva, Switzerland: 2013.

2. Green R, et al. Body iron excretion in man: a collaborative study. Am J Med. 1968; 45(3):336-53. [PubMed: 5672736]

3. Hallberg L, et al. Menstrual blood loss--a population study. Variation at different ages and attempts to define normality. Acta Obstet Gynecol Scand. 1966; 45(3):320-51. [PubMed: 5922481]

4. Zacharski LR, et al. Association of age, sex, and race with body iron stores in adults: analysis of NHANES III data. Am Heart J. 2000; 140(1):98-104. [PubMed: 10874269]

5. Zotter H, et al. Abnormally high serum ferritin levels among professional road cyclists. Br J Sports Med. 2004; 38(6):704-8. [PubMed: 15562163]

6. Lynch SR, Skikne BS, Cook JD. Food iron absorption in idiopathic hemochromatosis. Blood. 1989; 74(6):2187-93. [PubMed: 2804357]

7. Siddique A, Kowdley KV. Review article: the iron overload syndromes. Aliment Pharmacol Ther. 2012; 35(8):876-93. [PubMed: 22385471]

8. Fleming DJ, et al. Dietary determinants of iron stores in a free-living elderly population: The Framingham Heart Study. Am J Clin Nutr. 1998; 67(4):722-33. [PubMed: 9537620]

9. Milman N. Iron and pregnancy--a delicate balance. Ann Hematol. 2006; 85(9):559-65. [PubMed: 16691399]

10. Bardella MT, et al. Gluten intolerance: gender- and age-related differences in symptoms. Scand J Gastroenterol. 2005; 40(1):15-9. [PubMed: 15841709]

11. Schenck JF. Health and physiological effects of human exposure to whole-body four-tesla magnetic fields during MRI. Ann N Y Acad Sci. 1992; 649:285-301. [PubMed: 1580500]

12. Hentze MW, Muckenthaler MU, Andrews NC. Balancing acts: molecular control of mammalian iron metabolism. Cell. 2004; 117(3):285-97. [PubMed: 15109490]

13. Ahmad KA, et al. Decreased liver hepcidin expression in the Hfe knockout mouse. Blood Cells Mol Dis. 2002; 29(3):361-6. [PubMed: 12547226]

14. Nicolas G, et al. Severe iron deficiency anemia in transgenic mice expressing liver hepcidin. Proc Natl Acad Sci U S A. 2002; 99(7):4596-601. [PubMed: 11930010]

15. Park $\mathrm{CH}$, et al. Hepcidin, a urinary antimicrobial peptide synthesized in the liver. J Biol Chem. 2001; 276(11):7806-10. [PubMed: 11113131]

16. Pigeon $\mathrm{C}$, et al. A new mouse liver-specific gene, encoding a protein homologous to human antimicrobial peptide hepcidin, is overexpressed during iron overload. J Biol Chem. 2001; 276(11):7811-9. [PubMed: 11113132]

17. Nemeth E, et al. Hepcidin regulates cellular iron efflux by binding to ferroportin and inducing its internalization. Science. 2004; 306(5704):2090-3. [PubMed: 15514116]

18. Abboud S, Haile DJ. A novel mammalian iron-regulated protein involved in intracellular iron metabolism. J Biol Chem. 2000; 275(26):19906-12. [PubMed: 10747949]

19. Donovan A, et al. Positional cloning of zebrafish ferroportin1 identifies a conserved vertebrate iron exporter. Nature. 2000; 403(6771):776-81. [PubMed: 10693807]

20. McKie AT, et al. A novel duodenal iron-regulated transporter, IREG1, implicated in the basolateral transfer of iron to the circulation. Mol Cell. 2000; 5(2):299-309. [PubMed: 10882071]

21. Ganz T, Nemeth E. Hepcidin and disorders of iron metabolism. Annu Rev Med. 2011; 62:347-60. [PubMed: 20887198]

22. Nicolas G, et al. The gene encoding the iron regulatory peptide hepcidin is regulated by anemia, hypoxia, and inflammation. J Clin Invest. 2002; 110(7):1037-44. [PubMed: 12370282]

23. Milward EA, et al. Is HFE involved in increased hepcidin expression and hypoferremia in inflammation and anemia of chronic disease? Hepatology. 2005; 41(4):936-8. [PubMed: 15791616] 
24. Nemeth E, et al. IL-6 mediates hypoferremia of inflammation by inducing the synthesis of the iron regulatory hormone hepcidin. J Clin Invest. 2004; 113(9):1271-6. [PubMed: 15124018]

25. Andrews NC. Anemia of inflammation: the cytokine-hepcidin link. J Clin Invest. 2004; 113(9): 1251-3. [PubMed: 15124013]

26. Theurl I, et al. Regulation of iron homeostasis in anemia of chronic disease and iron deficiency anemia: diagnostic and therapeutic implications. Blood. 2009; 113(21):5277-86. [PubMed: 19293425]

27. Weiss G, Goodnough LT. Anemia of chronic disease. N Engl J Med. 2005; 352(10):1011-23. [PubMed: 15758012]

28. Martinelli N, et al. Increased serum hepcidin levels in subjects with the metabolic syndrome: a population study. PLoS One. 2012; 7(10):e48250. [PubMed: 23144745]

29. Kuragano T, et al. Hepcidin as well as TNF-alpha are significant predictors of arterial stiffness in patients on maintenance hemodialysis. Nephrol Dial Transplant. 2011; 26(8):2663-7. [PubMed: 21245128]

30. van der Weerd NC, et al. Hepcidin-25 in chronic hemodialysis patients is related to residual kidney function and not to treatment with erythropoiesis stimulating agents. PLoS One. 2012; 7(7):e39783. [PubMed: 22808058]

31. Galesloot TE, et al. Serum Hepcidin Is Associated With Presence of Plaque in Postmenopausal Women of a General Population. Arterioscler Thromb Vasc Biol. 2013

32. Hansson GK. Atherosclerosis--an immune disease: The Anitschkov Lecture 2007. Atherosclerosis. 2009; 202(1):2-10. [PubMed: 18951547]

33. Vaughan AM, Oram JF. ABCA1 and ABCG1 or ABCG4 act sequentially to remove cellular cholesterol and generate cholesterol-rich HDL. J Lipid Res. 2006; 47(11):2433-43. [PubMed: 16902247]

34. Tall AR. An overview of reverse cholesterol transport. Eur Heart J. 1998; 19(Suppl A):A31-5. [PubMed: 9519340]

35. Saeed O, et al. Pharmacological suppression of hepcidin increases macrophage cholesterol efflux and reduces foam cell formation and atherosclerosis. Arterioscler Thromb Vasc Biol. 2012; 32(2): 299-307. [PubMed: 22095982]

36. Koeth RA, et al. Intestinal microbiota metabolism of 1-carnitine, a nutrient in red meat, promotes atherosclerosis. Nat Med. Apr 7.2013 doi: 10.1038/nm.3145.

37. Nagy E, et al. Red cells, hemoglobin, heme, iron, and atherogenesis. Arterioscler Thromb Vasc Biol. 2010; 30(7):1347-53. [PubMed: 20378845]

38. Lipinski B, Pretorius E. Hydroxyl radical-modified fibrinogen as a marker of thrombosis: the role of iron. Hematology. 2012; 17(4):241-7. [PubMed: 22889519]

39. Shiffman D, et al. Large scale gene expression analysis of cholesterol-loaded macrophages. J Biol Chem. 2000; 275(48):37324-32. [PubMed: 10973959]

40. Chawla A, et al. A PPAR gamma-LXR-ABCA1 pathway in macrophages is involved in cholesterol efflux and atherogenesis. Mol Cell. 2001; 7(1):161-71. [PubMed: 11172721]

41. Chinetti G, et al. PPAR-alpha and PPAR-gamma activators induce cholesterol removal from human macrophage foam cells through stimulation of the ABCA1 pathway. Nat Med. 2001; 7(1): 53-8. [PubMed: 11135616]

42. Satchell L, Leake DS. Oxidation of low-density lipoprotein by iron at lysosomal pH: implications for atherosclerosis. Biochemistry. 2012; 51(18):3767-75. [PubMed: 22493939]

43. Bao W, et al. Dietary iron intake, body iron stores, and the risk of type 2 diabetes: a systematic review and meta-analysis. BMC Med. 2012; 10:119. [PubMed: 23046549]

44. Moreno PR, et al. Haptoglobin genotype is a major determinant of the amount of iron in the human atherosclerotic plaque. J Am Coll Cardiol. 2008; 52(13):1049-51. [PubMed: 18848136]

45. Pignoli $P$, et al. Intimal plus medial thickness of the arterial wall: a direct measurement with ultrasound imaging. Circulation. 1986; 74(6):1399-406. [PubMed: 3536154]

46. Staub D, et al. Contrast-enhanced ultrasound imaging of the vasa vasorum: from early atherosclerosis to the identification of unstable plaques. JACC Cardiovasc Imaging. 2010; 3(7): 761-71. [PubMed: 20633855] 
47. Cappendijk VC, et al. Assessment of human atherosclerotic carotid plaque components with multisequence MR imaging: initial experience. Radiology. 2005; 234(2):487-92. [PubMed: 15671004]

48. Puppini G, et al. Characterisation of carotid atherosclerotic plaque: comparison between magnetic resonance imaging and histology. Radiol Med. 2006; 111(7):921-30. [PubMed: 17021689]

49. Albuquerque LC, et al. Intraplaque hemorrhage assessed by high-resolution magnetic resonance imaging and C-reactive protein in carotid atherosclerosis. J Vasc Surg. 2007; 46(6):1130-7. [PubMed: 17920229]

50. Mitsumori LM, et al. In vivo accuracy of multisequence MR imaging for identifying unstable fibrous caps in advanced human carotid plaques. J Magn Reson Imaging. 2003; 17(4):410-20. [PubMed: 12655579]

51. Young VE, et al. Diffusion-weighted magnetic resonance imaging for the detection of lipid-rich necrotic core in carotid atheroma in vivo. Neuroradiology. 2010; 52(10):929-36. [PubMed: 20358366]

52. Moody AR, et al. Characterization of complicated carotid plaque with magnetic resonance direct thrombus imaging in patients with cerebral ischemia. Circulation. 2003; 107(24):3047-52. [PubMed: 12796133]

53. Qiao Y, et al. Identification of intraplaque hemorrhage on MR angiography images: a comparison of contrast-enhanced mask and time-of-flight techniques. AJNR Am J Neuroradiol. 2011; 32(3): 454-9. [PubMed: 21233234]

54. Cai JM, et al. Classification of human carotid atherosclerotic lesions with in vivo multicontrast magnetic resonance imaging. Circulation. 2002; 106(11):1368-73. [PubMed: 12221054]

55. Saam T, et al. Quantitative evaluation of carotid plaque composition by in vivo MRI. Arterioscler Thromb Vasc Biol. 2005; 25(1):234-9. [PubMed: 15528475]

56. Hatsukami TS, et al. Visualization of fibrous cap thickness and rupture in human atherosclerotic carotid plaque in vivo with high-resolution magnetic resonance imaging. Circulation. 2000; 102(9):959-64. [PubMed: 10961958]

57. Cappendijk VC, et al. Comparison of lipid-rich necrotic core size in symptomatic and asymptomatic carotid atherosclerotic plaque: Initial results. J Magn Reson Imaging. 2008; 27(6): 1356-61. [PubMed: 18504755]

58. Yuan C, et al. In vivo accuracy of multispectral magnetic resonance imaging for identifying lipidrich necrotic cores and intraplaque hemorrhage in advanced human carotid plaques. Circulation. 2001; 104(17):2051-6. [PubMed: 11673345]

59. Bitar R, et al. In vivo 3D high-spatial-resolution MR imaging of intraplaque hemorrhage. Radiology. 2008; 249(1):259-67. [PubMed: 18796681]

60. Chu B, et al. Hemorrhage in the atherosclerotic carotid plaque: a high-resolution MRI study. Stroke. 2004; 35(5):1079-84. [PubMed: 15060318]

61. Kampschulte A, et al. Differentiation of intraplaque versus juxtaluminal hemorrhage/thrombus in advanced human carotid atherosclerotic lesions by in vivo magnetic resonance imaging. Circulation. 2004; 110(20):3239-44. [PubMed: 15533871]

62. Ota $\mathrm{H}$, et al. Carotid intraplaque hemorrhage imaging at 3.0-T MR imaging: comparison of the diagnostic performance of three T1-weighted sequences. Radiology. 2010; 254(2):551-63. [PubMed: 20093526]

63. Yim YJ, et al. High signal intensity halo around the carotid artery on maximum intensity projection images of time-of-flight MR angiography: a new sign for intraplaque hemorrhage. J Magn Reson Imaging. 2008; 27(6):1341-6. [PubMed: 18504753]

64. Lima JA, et al. Statin-induced cholesterol lowering and plaque regression after 6 months of magnetic resonance imaging-monitored therapy. Circulation. 2004; 110(16):2336-41. [PubMed: 15477398]

65. West AM, et al. Low-density lipoprotein lowering does not improve calf muscle perfusion, energetics, or exercise performance in peripheral arterial disease. J Am Coll Cardiol. 2011; 58(10): 1068-76. [PubMed: 21867844]

66. Anderson LJ, et al. Cardiovascular T2-star (T2*) magnetic resonance for the early diagnosis of myocardial iron overload. Eur Heart J. 2001; 22(23):2171-9. [PubMed: 11913479] 
67. Tanner MA, et al. Multi-center validation of the transferability of the magnetic resonance T2* technique for the quantification of tissue iron. Haematologica. 2006; 91(10):1388-91. [PubMed: 17018390]

68. Westwood M, et al. A single breath-hold multiecho T2* cardiovascular magnetic resonance technique for diagnosis of myocardial iron overload. J Magn Reson Imaging. 2003; 18(1):33-9. [PubMed: 12815637]

69. Modell B, et al. Improved survival of thalassaemia major in the UK and relation to T2* cardiovascular magnetic resonance. J Cardiovasc Magn Reson. 2008; 10:42. [PubMed: 18817553]

70. Chavhan GB, et al. Principles, techniques, and applications of T2*-based MR imaging and its special applications. Radiographics. 2009; 29(5):1433-49. [PubMed: 19755604]

71. Shoden A, Sturgeon P. Hemosiderin. Acta Haematologica. 1960; 23(6):376-392.

72. Diamagnetic Susceptibility of Organic Compounds, Oils, Paraffins and Polyethylenes. Springer; 2008.

73. Sharkey-Toppen TP, et al. Improved in vivo human carotid artery wall T(2) estimation. Magn Reson Imaging. 2013; 31(1):44-52. [PubMed: 22921942]

74. Kirk P, et al. International reproducibility of single breathhold T2* MR for cardiac and liver iron assessment among five thalassemia centers. J Magn Reson Imaging. 2010; 32(2):315-9. [PubMed: 20677256]

75. Kolodgie FD, et al. Intraplaque hemorrhage and progression of coronary atheroma. N Engl J Med. 2003; 349(24):2316-25. [PubMed: 14668457]

76. Raman SV, et al. In vivo atherosclerotic plaque characterization using magnetic susceptibility distinguishes symptom-producing plaques. JACC Cardiovasc Imaging. 2008; 1(1):49-57. [PubMed: 19356405]

77. Howarth SP, et al. In vivo positive contrast IRON sequence and quantitative $\mathrm{T}(2)^{*}$ measurement confirms inflammatory burden in a patient with asymptomatic carotid atheroma after USPIOenhanced MR imaging. J Vasc Interv Radiol. 2008; 19(3):446-8. [PubMed: 18295707]

78. Patterson AJ, et al. In vivo carotid plaque MRI using quantitative T2* measurements with ultrasmall superparamagnetic iron oxide particles: a dose-response study to statin therapy. NMR Biomed. 2011; 24(1):89-95. [PubMed: 21259368]

79. Ludwiczek $\mathrm{S}$, et al. Cytokine-mediated regulation of iron transport in human monocytic cells. Blood. 2003; 101(10):4148-54. [PubMed: 12522003]

80. Recalcati S, et al. Response of monocyte iron regulatory protein activity to inflammation: abnormal behavior in genetic hemochromatosis. Blood. 1998; 91(7):2565-72. [PubMed: 9516158]

81. Schroeder $\mathrm{S}$, et al. Noninvasive detection and evaluation of atherosclerotic coronary plaques with multislice computed tomography. J Am Coll Cardiol. 2001; 37(5):1430-5. [PubMed: 11300457]

82. Papadopoulou SL, et al. Detection and quantification of coronary atherosclerotic plaque by 64-slice multidetector CT: a systematic head-to-head comparison with intravascular ultrasound. Atherosclerosis. 2011; 219(1):163-70. [PubMed: 21802687]

83. Voros $\mathrm{S}$, et al. Coronary atherosclerosis imaging by coronary CT angiography: current status, correlation with intravascular interrogation and meta-analysis. JACC Cardiovasc Imaging. 2011; 4(5):537-48. [PubMed: 21565743]

84. Pletcher MJ, et al. Interpretation of the coronary artery calcium score in combination with conventional cardiovascular risk factors: the Multi-Ethnic Study of Atherosclerosis (MESA). Circulation. 2013; 128(10):1076-84. [PubMed: 23884352]

85 . Polonsky TS, et al. Coronary artery calcium score and risk classification for coronary heart disease prediction. JAMA. 2010; 303(16):1610-6. [PubMed: 20424251]

86. Hoffmann U, et al. Noninvasive assessment of plaque morphology and composition in culprit and stable lesions in acute coronary syndrome and stable lesions in stable angina by multidetector computed tomography. J Am Coll Cardiol. 2006; 47(8):1655-62. [PubMed: 16631006]

87. Rajendran R, et al. Does iron inhibit calcification during atherosclerosis? Free Radic Biol Med. 2012; 53(9):1675-9. [PubMed: 22940067]

88. Karolyi M, et al. Classification of coronary atherosclerotic plaques ex vivo with T1, T2, and ultrashort echo time CMR. JACC Cardiovasc Imaging. 2013; 6(4):466-74. [PubMed: 23498670] 
89. Wu Z, et al. Identification of calcification with MRI using susceptibility-weighted imaging: a case study. J Magn Reson Imaging. 2009; 29(1):177-82. [PubMed: 19097156]

90. Greenland P, et al. 2010 ACCF/AHA guideline for assessment of cardiovascular risk in asymptomatic adults: a report of the American College of Cardiology Foundation/American Heart Association Task Force on Practice Guidelines. J Am Coll Cardiol. 2010; 56(25):e50-103. [PubMed: 21144964]

91. Mani V, et al. Carotid black blood MRI burden of atherosclerotic disease assessment correlates with ultrasound intima-media thickness. J Cardiovasc Magn Reson. 2006; 8(3):529-34. [PubMed: 16755842]

92. Baldassarre D, et al. Carotid intima-media thickness and markers of inflammation, endothelial damage and hemostasis. Ann Med. 2008; 40(1):21-44. [PubMed: 17934910]

93. Kastelein JJ, et al. Simvastatin with or without ezetimibe in familial hypercholesterolemia. N Engl J Med. 2008; 358(14):1431-43. [PubMed: 18376000]

94. Brott TG, et al. 2011 ASA/ACCF/AHA/AANN/AANS/ACR/ASNR/CNS/SAIP/SCAI/SIR/ SNIS/SVM/SVS Guideline on the Management of Patients With Extracranial Carotid and Vertebral Artery Disease: Executive Summary A Report of the American College of Cardiology Foundation/American Heart Association Task Force on Practice Guidelines, and the American Stroke Association, American Association of Neuroscience Nurses, American Association of Neurological Surgeons, American College of Radiology, American Society of Neuroradiology, Congress of Neurological Surgeons, Society of Atherosclerosis Imaging and Prevention, Society for Cardiovascular Angiography and Interventions, Society of Interventional Radiology, Society of NeuroInterventional Surgery, Society for Vascular Medicine, and Society for Vascular Surgery Developed in Collaboration With the American Academy of Neurology and Society of Cardiovascular Computed Tomography. J Am Coll Cardiol. 2011; 57(8):1002-44. [PubMed: 21288680]

95. Gerhard-Herman M, et al. Guidelines for noninvasive vascular laboratory testing: a report from the American Society of Echocardiography and the Society for Vascular Medicine and Biology. Vasc Med. 2006; 11(3):183-200. [PubMed: 17288127]

96. Takaya N, et al. Association between carotid plaque characteristics and subsequent ischemic cerebrovascular events: a prospective assessment with MRI--initial results. Stroke. 2006; 37(3): 818-23. [PubMed: 16469957]

97. Giroud D, et al. Relation of the site of acute myocardial infarction to the most severe coronary arterial stenosis at prior angiography. Am J Cardiol. 1992; 69(8):729-32. [PubMed: 1546645]

98. Chang CC, et al. Simvastatin downregulates the expression of hepcidin and erythropoietin in HepG2 cells. Hemodial Int. 2013; 17(1):116-21. [PubMed: 22716163]

99. Zacharski LR, et al. Reduction of iron stores and cardiovascular outcomes in patients with peripheral arterial disease: a randomized controlled trial. JAMA. 2007; 297(6):603-10. [PubMed: 17299195]

100. Zacharski LR, et al. The statin-iron nexus: anti-inflammatory intervention for arterial disease prevention. Am J Public Health. 2013; 103(4):e105-12. [PubMed: 23409890]

101. Minqin R, et al. The iron chelator desferrioxamine inhibits atherosclerotic lesion development and decreases lesion iron concentrations in the cholesterol-fed rabbit. Free Radic Biol Med. 2005; 38(9):1206-11. [PubMed: 15808418]

102. Duffy SJ, et al. Iron chelation improves endothelial function in patients with coronary artery disease. Circulation. 2001; 103(23):2799-804. [PubMed: 11401935]

103. Lamas GA, et al. Effect of disodium EDTA chelation regimen on cardiovascular events in patients with previous myocardial infarction: the TACT randomized trial. JAMA. 2013; 309(12): 1241-50. [PubMed: 23532240]

104. Araujo JA, Zhang M, Yin F. Heme oxygenase-1, oxidation, inflammation, and atherosclerosis. Front Pharmacol. 2012; 3:119. [PubMed: 22833723]

105. Cooke KS, et al. A fully human anti-hepcidin antibody modulates iron metabolism in both mice and nonhuman primates. Blood. 2013; 122(17):3054-61. [PubMed: 23945155]

106. Danesh J, Appleby P. Coronary heart disease and iron status: meta-analyses of prospective studies. Circulation. 1999; 99(7):852-4. [PubMed: 10027804] 


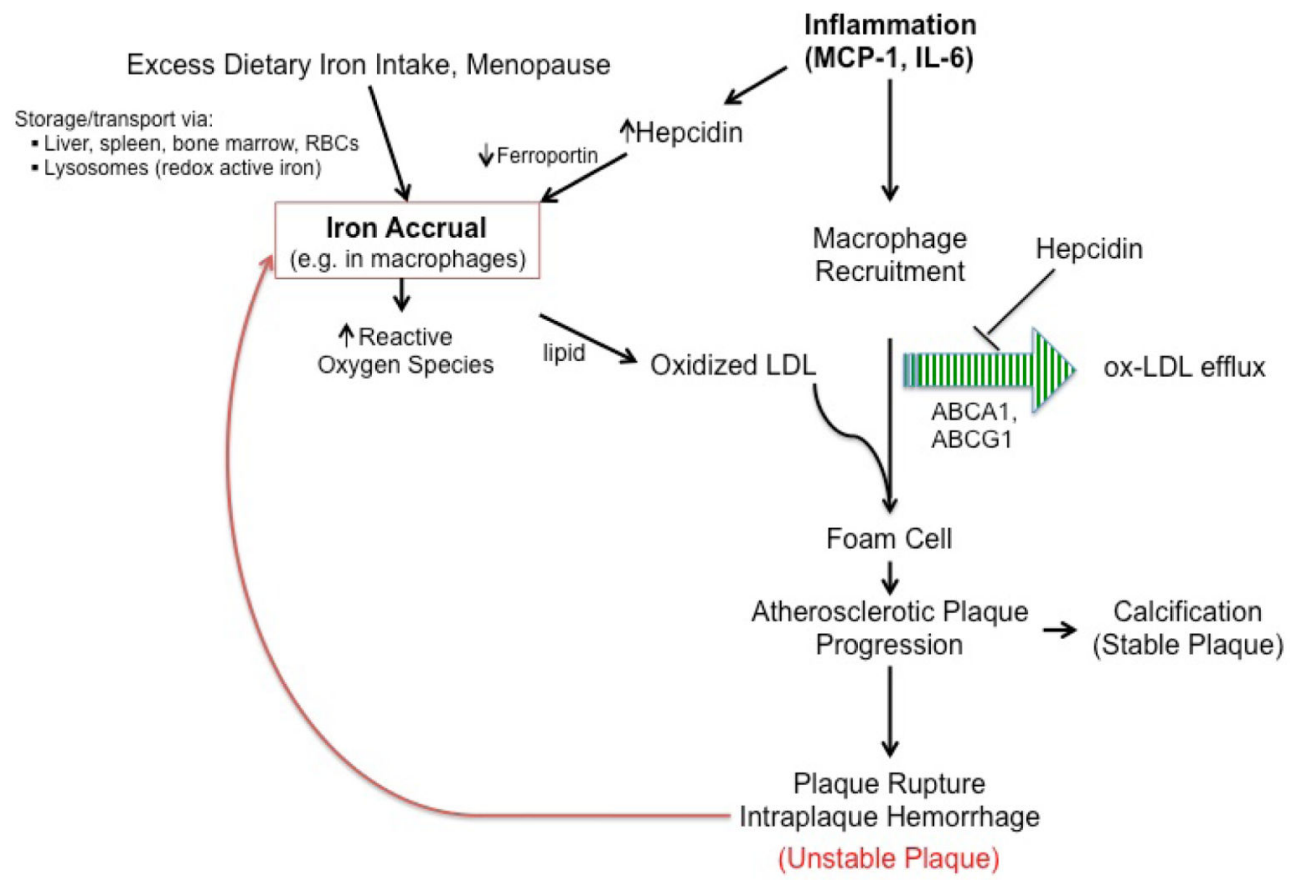

Figure 1.

This schematic depicts various pathways that indicate iron's role in atherosclerosis initiation and progression. 


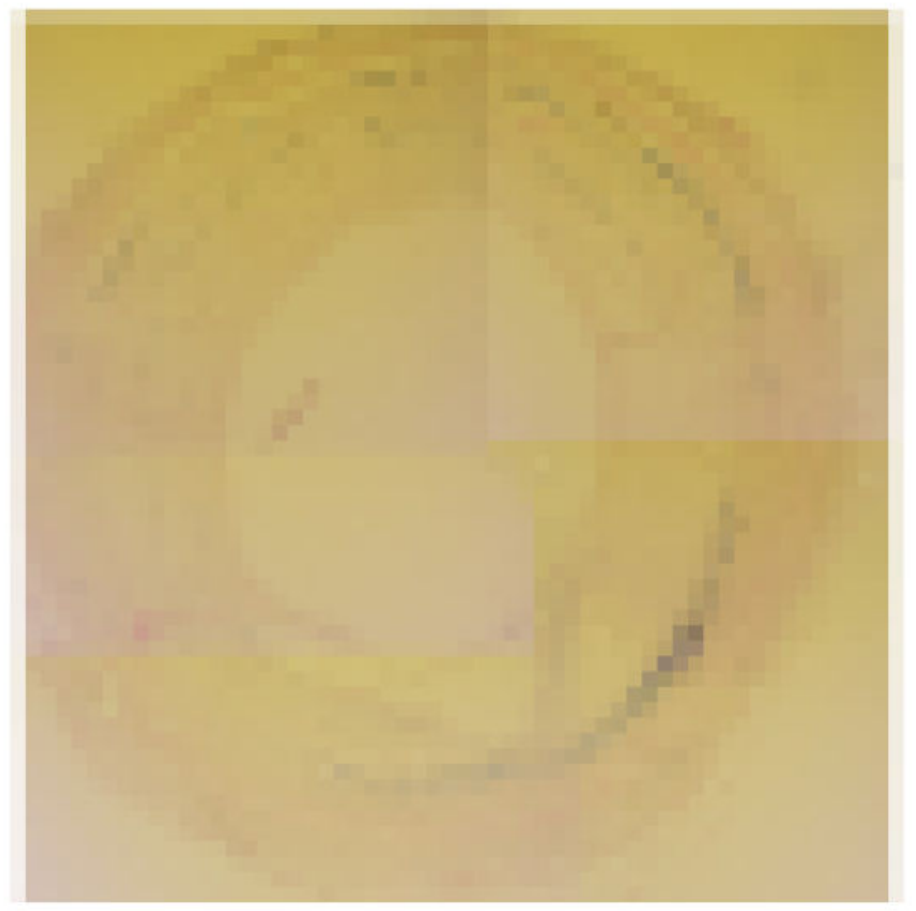

Figure 2.

Low power light microscopy of an autopsy human coronary artery section stained with Prussian blue. Note the near-circumferential staining indicating the presence of iron in the arterial wall. 

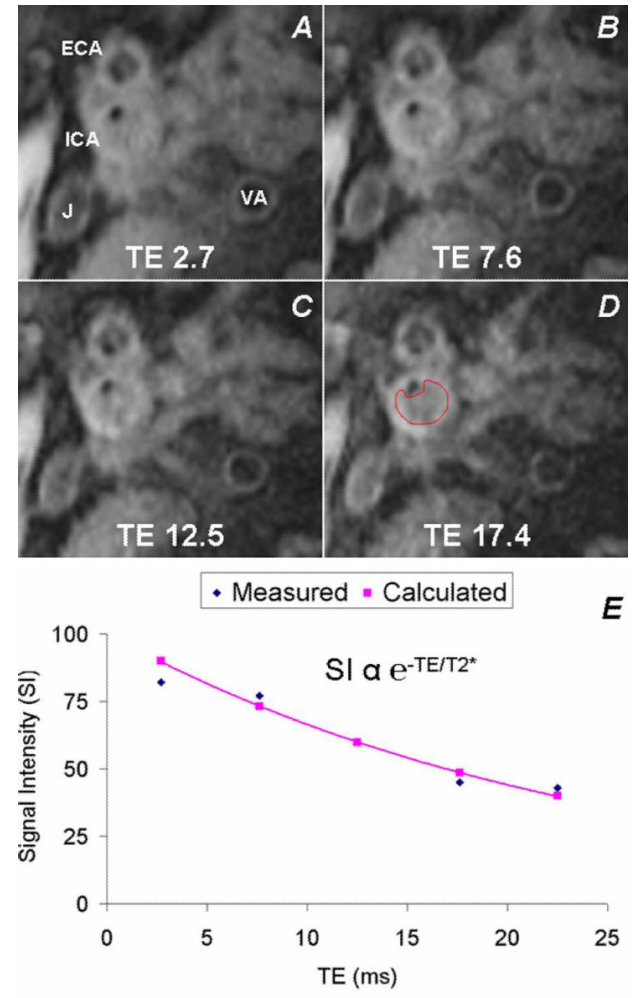

Figure 3.

Magnetic resonance imaging (MRI) can, without the need for exogenous contrast agent, quantify tissue iron content using a technique called T2* mapping. This is based on the exponential signal intensity decay over subsequently longer echo times (TE) that occurs more rapidly in tissues with increased iron. Shown are MRI images acquired in an orthogonal plane through an atherosclerotic internal carotid artery (ICA) at serial TEs from which intraplaque $\mathrm{T} 2 *$ can be calculated. $\mathrm{ECA}=$ external carotid artery, $\mathrm{V}=$ vertebral artery, $\mathrm{J}=$ jugular vein. Reproduced with permission from JACC: Cardiovascular Imaging. 


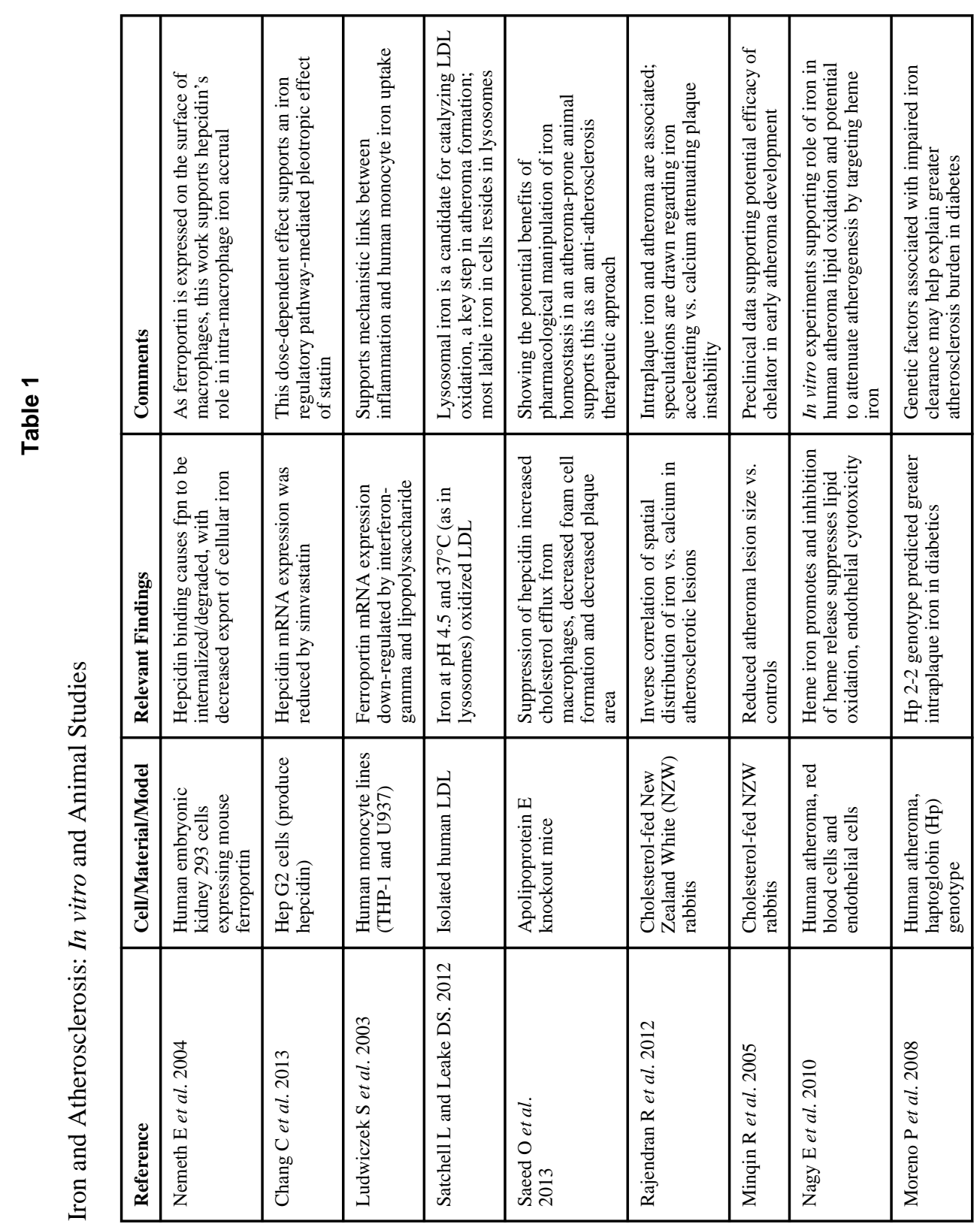

J Cardiovasc Transl Res. Author manuscript; available in PMC 2015 July 01. 


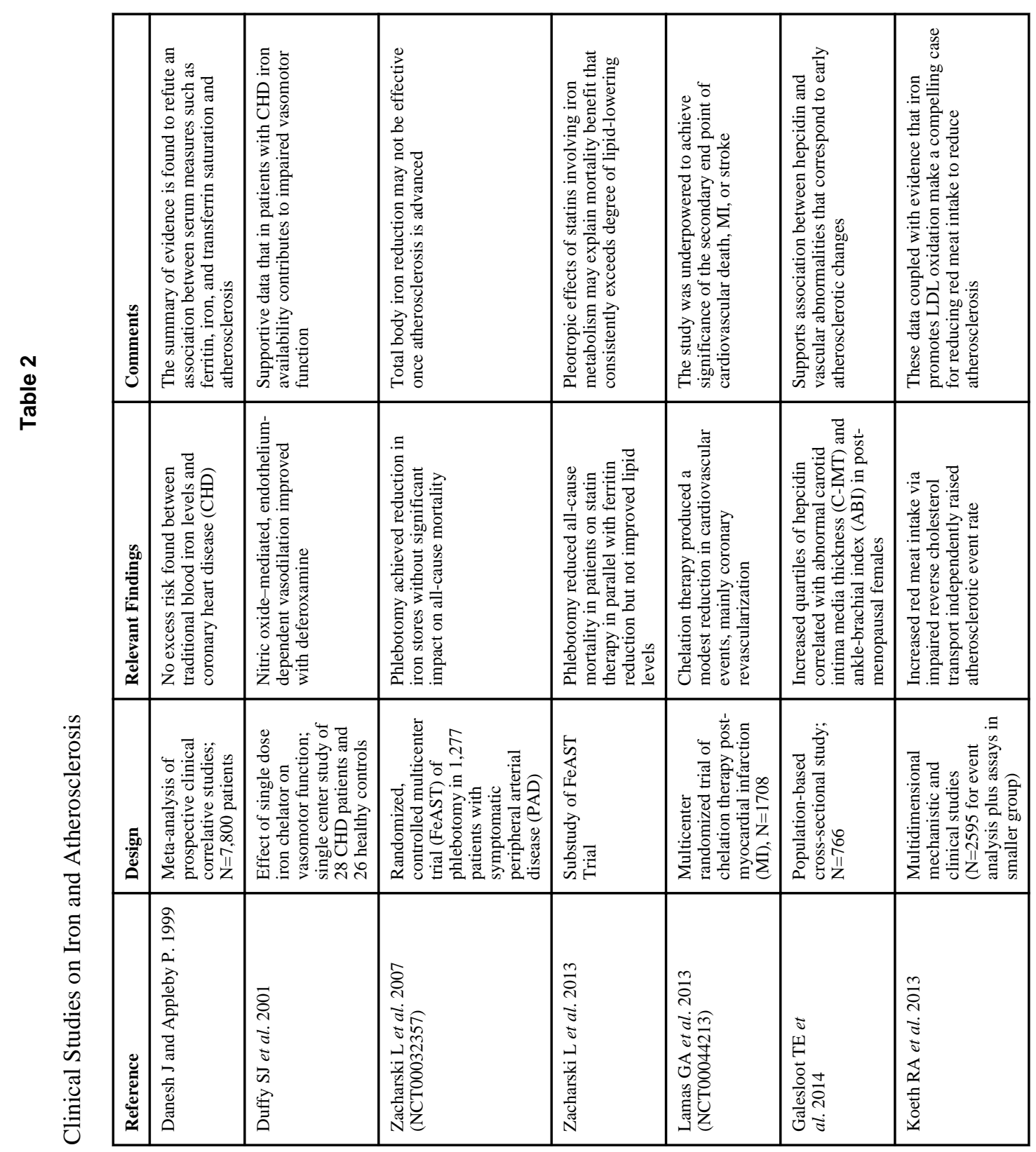

J Cardiovasc Transl Res. Author manuscript; available in PMC 2015 July 01. 